\title{
Human motor cortex activity during mental rotation
}

\author{
Christian Windischberger, ${ }^{\mathrm{a}}$ Claus Lamm, ${ }^{\mathrm{b}}$ Herbert Bauer, ${ }^{\mathrm{b}}$ and Ewald Moser ${ }^{\mathrm{a}, \mathrm{c}, *}$ \\ ${ }^{a}$ NMR Group, Department of Medical Physics, University of Vienna, Währingerstrasse 13, A-1090 Vienna, Austria \\ ${ }^{\mathrm{b}}$ Brain Research Lab, Department of Psychology, University of Vienna, Liebiggasse 5, A-1010 Vienna, Austria \\ ${ }^{\mathrm{c}}$ Department of Radiodiagnostics, AKH and University of Vienna, Währinger Gürtel 18-20, A-1090 Vienna, Austria
}

Received 13 September 2002; revised 13 March 2003; accepted 21 April 2003

\begin{abstract}
The functional role of human premotor and primary motor cortex during mental rotation has been studied using functional MRI at 3 T. Fourteen young, male subjects performed a mental rotation task in which they had to decide whether two visually presented cubes could be identical. Exploratory Fuzzy Cluster Analysis was applied to identify brain regions with stimulus-related time courses. This revealed one dominant cluster which included the parietal cortex, premotor cortex, and dorsolateral prefrontal cortex that showed signal enhancement during the whole stimulus presentation period, reflecting cognitive processing. A second cluster, encompassing the contralateral primary motor cortex, showed activation exclusively after the button press response. This clear separation was possible in 3 subjects only, however. Based on these exploratory results, the hypothesis that primary motor cortex activity was related to button pressing only was tested using a parametric approach via a random-effects group analysis over all 14 subjects in SPM99. The results confirmed that the stimulus response via button pressing causes activation in the primary motor cortex and supplementary motor area while parietal cortex and mesial regions rostral to the supplementary motor area are recruited for the actual mental rotation process.

(C) 2003 Elsevier Science (USA). All rights reserved.
\end{abstract}

\section{Introduction}

The nature of mental imagery has long been the subject of neuroscience research (Kosslyn, 1994). Shepard and Metzler were among the first researchers to investigate the process of mental imagery. In their famous behavioral experiment (Shepard and Metzler, 1971) they presented two geometric objects at different orientations to subjects and asked whether these objects were identical or mirror copies of each other. Remarkably, the subjects' response times increased almost linearly with the angular difference between the two objects. From these results it was hypothesized that subjects "mentally rotated" the mental image of one object along a trajectory until it had the same orientation as the other object and then compared both for congruency. Based on this study, a number of experiments investigating the brain responses underlying mental rotation processes have been performed, using electrophysiological methods, positron emission tomography and functional

* Corresponding author. Fax: +43-1-4277-9607.

E-mail address: ewald.moser@univie.ac.at (E. Moser). magnetic resonance imaging (fMRI) (Cohen et al., 1996; Alivisatos and Petrides, 1997; Tagaris et al., 1997, 1998; Richter et al., 1997, 2000; Kosslyn et al., 1998; Carpenter et al., 1999; Harris et al., 2000; Yoshino et al., 2000; Jordan et al., 2001; Lamm et al., 2001; Vingerhoets et al., 2001, 2002). Although it is generally agreed that the parietal cortex is involved in the mental rotation process, there is considerable disagreement about interhemispheric activation differences. For Shepard-Metzler-like stimuli most studies have reported bilateral parietal activation (Cohen et al., 1996; Richter et al., 1997, 2000; Jordan et al., 2001), while experiments using other stimuli demonstrated predominance of either the left (Alivisatos and Petrides, 1997) or the right hemisphere (Harris et al., 2000; Yoshino et al., 2000) or no distinct difference (Tagaris et al., 1998; Lamm et al., 2001).

Even more disagreement is apparent in the literature with respect to the question of whether motor areas are involved in the actual mental rotation process. Theoretical concepts suggest that dynamic imagery and overt movements rely on the same neural networks, i.e., they are functionally equivalent (Prinz, 1997; Weimer, 1997). In a recent study, Wex- 
ler et al. showed that subjects performing a mental rotation task and an unseen rotation in a given direction exhibited shorter reaction times and fewer errors when the motor rotation direction was compatible with the mental rotation direction (Wexler et al., 1998). The authors concluded that mental rotation would recruit motor planning and anticipation, but not the cortical and subcortical mechanisms responsible for movement execution. Imaging studies have, however, shown inconclusive results. Some found activity in the premotor (PM) cortex (Cohen et al., 1996; Richter et al., 2000), while others did not report PM involvement during mental rotation of both two-dimensional and threedimensional objects (Kosslyn, et al., 1998; Harris et al., 2000; Jordan et al., 2001). Concerning the primary motor cortex, Richter et al. (2000) reported that activation in contralateral primary motor (M1) cortex seems to be related to the button press at the end of each task, but could not rule out that M1 is also involved in another aspect of the task. Recently, in a mental rotation study using pictures of hands and tools as stimuli, Vingerhoets et al. did not find activation in the primary motor cortex (Vingerhoets et al., 2002). However, they suggested that M1 is indeed active during mental rotation, but less prominently than premotor areas and is therefore often not visible due to "methodological noise."

The majority of these studies have used model-dependent methods for fMRI analysis, e.g., the general linear model as implemented in SPM (http://www.fil.ion.ac.uk/ spm; Friston et al., 1995). These techniques apply reference or basis functions that are derived from the stimulus paradigm and try to determine the extent to which voxel time courses fit the model. Intrinsically, model-based analyses are as good as the model they are based on. There are also a number of exploratory data analysis (EDA) methods applied in fMRI, such as Fuzzy Custer Analysis (FCA) (Scarth et al., 1995; Moser et al., 1997, 1999), Principal Component Analysis (Sychra et al., 1994; Backfrieder et al., 1996), Independent Component Analysis (McKeown et al., 1998; Calhoun et al., 2001), or Hierarchical Clustering (Filzmoser et al., 1999; Goutte et al., 1999). An intention common to all EDA techniques is to minimize the need for information on the nature and/or the timing of the stimuli. Rather, they attempt to express the large number of time courses present in fMRI data sets with a small number of clusters or components. The results of an EDA contain information on all "representative" time courses and, as such, are independent of the underlying model assumptions. Given a specific stimulus presentation paradigm, it is possible to use these results to formulate a hypothesis of the actual underlying psychological and physiological occurrences. In this study we have applied paradigm-free FCA to data from a mental rotation paradigm to examine the role of PM and M1 during mental rotation. Based on those FCA results we have set up the hypothesis that M1 is active only while responding but not during the mental rotation task proper. To statistically test this hypothesis suggested by exploratory FCA, data sets of all 14 subjects also underwent a random-effects group analysis in SPM using regressors that were based on the FCA results.

\section{Materials and methods}

\section{Subjects and task paradigm}

We examined 14 young, healthy, male subjects (age range 19-28) with no known history of neurological disorders. Written informed consent was obtained from all subjects prior to measurements and the study was approved by the local ethics committee. All participants were righthanded according to the Marian Annett handedness inventory (Annett, 1985). Tasks were derived from a standardized cube comparison test (3-dimensional cube test, 3DC, Gittler, 1990). Since these fulfill the criteria of the Rasch model (Rasch, 1980), they have the property of unidimensionality. This means that 3DC tasks measure one and the same latent cognitive dimension (ability) in different groups of subjects. Two runs of $15 \mathrm{~min}$ each were measured. The stimuli were presented to the subjects via MR compatible video goggles (Resonance Technologies, Los Angeles, CA, USA). Each run started with a baseline (BL) image, consisting of two blue-filled cubes on a black background with a white fixation cross in between them. After $20 \mathrm{~s}$ of BL images the empty cube faces were replaced with white patterns (parallel lines, triangle, etc.; Fig. 1), and the subjects had to decide whether the two cubes could be identical or not (under the assumptions that each pattern exists only once on each cube). This required the mental rotation/transformation of one of the cubes and a matching to the other. Subjects answered by pressing a button with their right hands. As task presentation time was not limited, the number of trials answered varied across subjects in a range from 27 to 50 (for both runs). All MRI experiments were performed on a 3-T Medspec S300 whole-body system (Bruker Biospin, Ettlingen, Germany) using a whole-body gradient system and a standard birdcage coil for RF excitation/reception. We used a single-shot, blipped gradient-recalled EPI sequence with a matrix size of 64 by 64 pixels and an TE of $23 \mathrm{~ms}$ (Windischberger and Moser, 2000). We acquired 15 axial slices with a FOV of 190 by $190 \mathrm{~mm}$, a thickness of $5 \mathrm{~mm}$, and an interslice distance of $1 \mathrm{~mm}$, covering nearly the whole cerebrum. Repetition time for the whole image slab was $1.5 \mathrm{~s}$.

\section{Data analysis}

All fMRI data were realigned to the first image using AIR v3.08 (Woods et al., 1998). The motion-corrected data sets were analyzed on an exploratory basis using FCA implemented in the software package EvIdent (http:// www.ibd.nrc.ca/evident; Scarth et al., 1995; Moser et al., 1997, 1999; Baumgartner et al., 1998). FCA represents a 


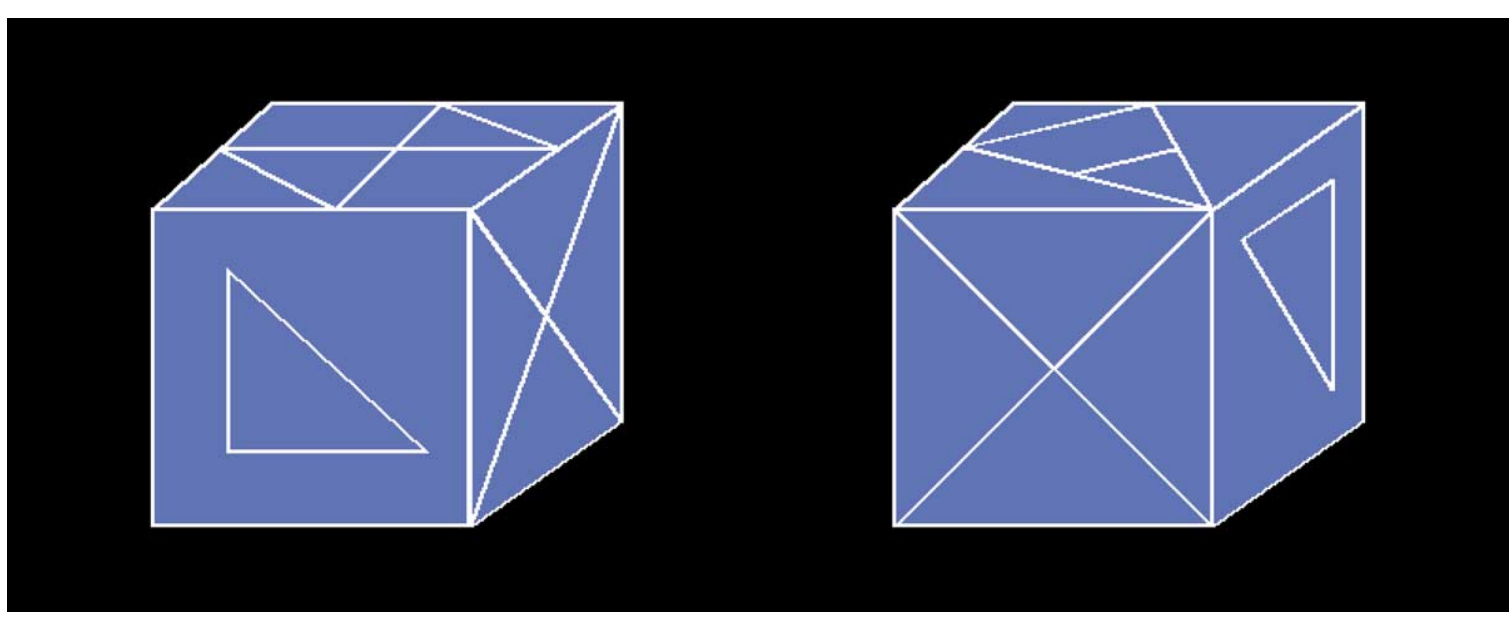

Fig. 1. Sample 3D cube comparison stimuli.

paradigm-free analysis method as it does not require explicit knowledge of the stimulus timing used during the experiment, but searches for similar pixel time courses and groups such pixels into clusters. These clusters (i.e., pixel maps and their corresponding mean time courses) are returned as results, and it is the task of the experimenter to assess the significance and relevance of the individual clusters. The two 15-min runs acquired per subject were examined separately. A two-step clustering strategy was used. In the first clustering step, all pixels with relevance to the paradigm were extracted. These pixels were then subject to a second cluster analysis, where more subtle temporal features were examined (e.g., activation amplitude).

We took advantage of a pixel preselection feature implemented in EvIdent in which the frequency spectrum of each pixel time course is determined and a restriction of the initial FCA to certain frequency components is allowed. This is of particular importance in event-related fMRI as at least an estimate of the number of stimuli applied is known in advance. It should be noted that this preselection does not exclude the other pixels, but rather improves cluster initalization, as all nonpreselected pixels are added in the last clustering iteration and are assigned to the clusters according to their temporal features.

From the results of the FCA we found distinct pixel groups that showed activation during the whole stimulus presentation period, while others exhibited strong, shorttime signal enhancements after task completion (see Results). It appears that M1 is not used for the actual mental transformation process but rather a consequence of responding via button press.

In order to statistically test these properties we performed an analysis in SPM99 (Friston et al., 1995) with two reference functions. The regressor that corresponded to cognitive task processing was modeled as a sequence of epochs according to the stimulus processing times obtained during the experiment. A second regressor representing task response was constructed as events at the response times with a length of 1 TR each. Both regressors were convolved with the hemodynamic response function as implemented in the SPM package. An example of the regressors created is shown in Fig. 4b together with the corresponding FCA results (Fig. 4a). For the group analysis, all data sets were normalized to the MNI standard brain and were smoothed with an isotropic Gaussian kernel of $9 \mathrm{~mm}$ full-width-athalf-maximum. The statistical maps were then used for a random-effects analysis including all 14 subjects.

\section{Results}

\section{FCA results}

FCA was able to detect stimulus-related time courses in both runs in each of the 14 subjects. A clear distinction of cognitive and motor clusters was possible in 3 subjects only, however. Fig. 2 shows the activation maps of two clusters resulting from the second clustering step for a single subject. The corresponding time courses in terms of cluster centroids are given in Fig. 3, where stimulus presentation periods are indicated below. Both centroids exhibit a sequence of peaks that correspond to the stimuli presented. Cluster 1 (Fig. 2a) contains pixels in the parietal, occipital, and premotor as well as dorsolateral prefrontal cortex and supplementary motor area (SMA).

The time course of cluster 1 (Fig. 3a) follows the stimulation paradigm very closely. Similarly, the centroid of cluster 2 (Fig. 3b) exhibits stimulus correspondence, but in this case the signal enhancement occurs after the end of the stimulus presentation period and shows a more peak-like shape. Fig. 4 shows an enlarged section of the time courses of both clusters, together with the stimulation paradigm. Centroid differences are even more apparent in this case. From the pixel overlays (Fig. 2) it can be seen that cluster 2 contains a small number of pixels only and these pixels are located primarily in the primary motor cortex (M1; 
(a)
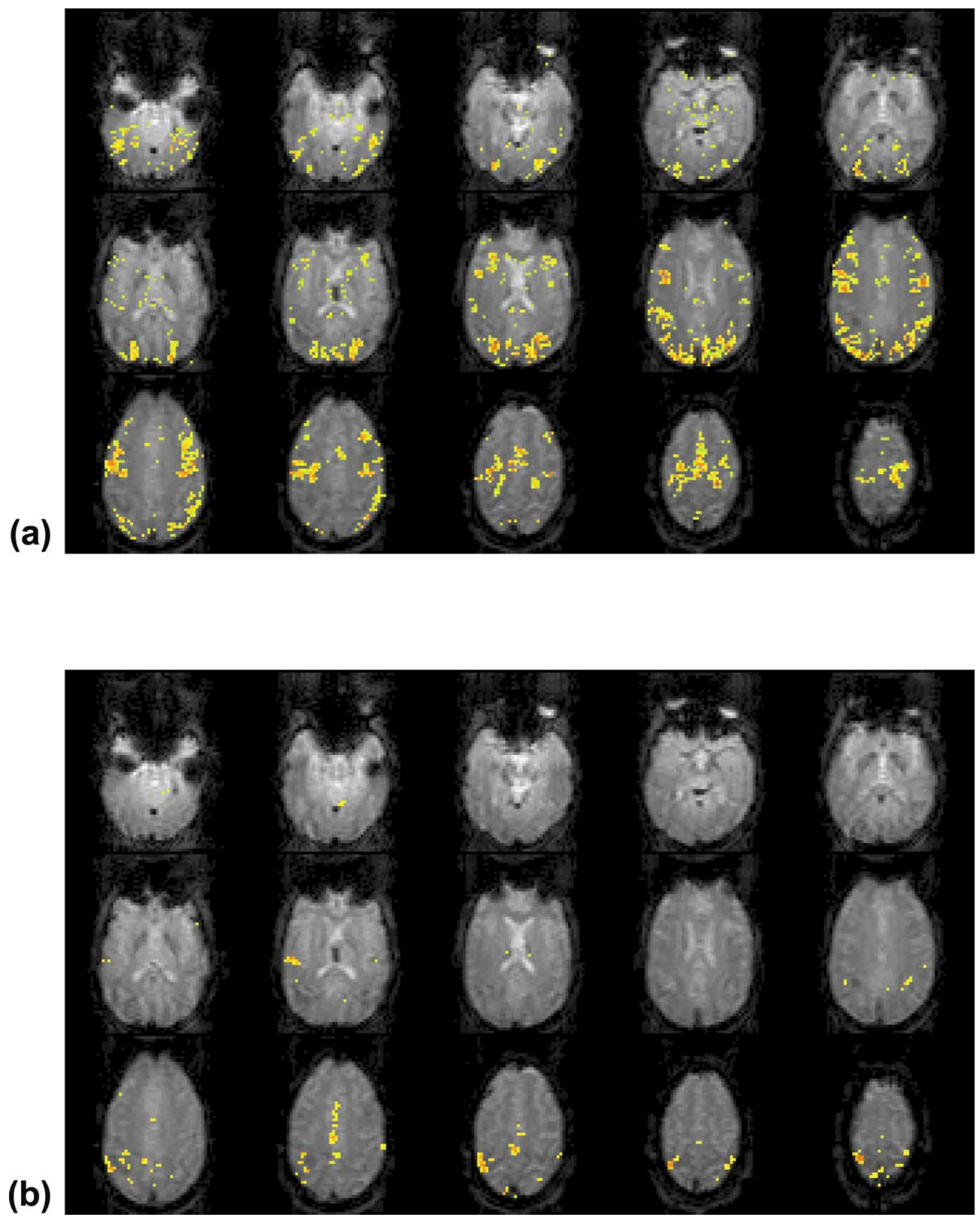

Fig. 2. FCA results for one subject. (a) Cluster 1 showing activation due to cognitive processing in parietal and premotor areas; (b) cluster 2 containing focal activation in the primary motor cortex caused by response via button pressing.

contralateral to the hand that entered the task response), as well as in the SMA. Both the time course and the activation maps suggest that the activation in cluster 2 is not related to mental imagery as required for task solving, but rather reflects motor activity due to answering (button pressing). In other words, these results strongly indicate that primary motor cortex is not used during mental rotation of 3DC stimuli. 


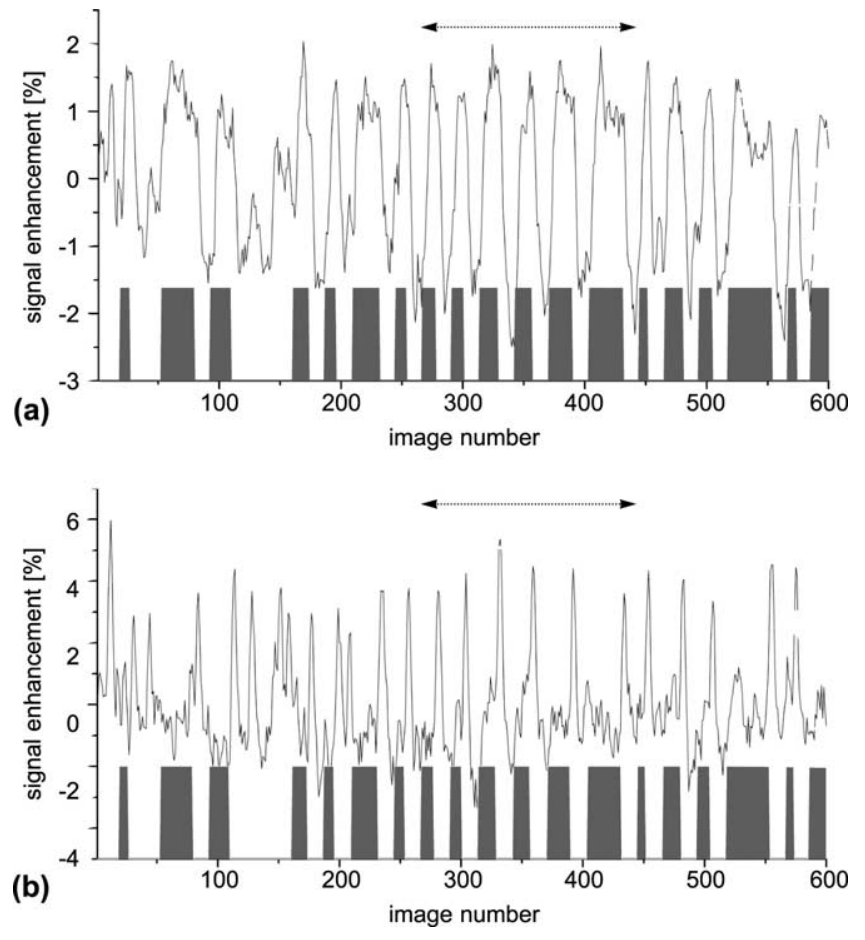

Fig. 3. Time courses of activated pixel from FCA results (Fig. 2) for cluster 1 (a) and cluster 2 (b). Mental rotation trials are indicated by gray blocks. Note that cluster 1 is activated throughout the stimulation period while cluster 2 shows brain activity at response times only.

\section{SPM results}

As only three subjects showed a clear separation of cognitive and motor activation in the FCA results, we sought to statistically test the involvement of M1 in visuospatial processing via random-effects group analysis in the parametric approach of SPM.

To allow a group analysis, all data sets were normalized to the MNI brain and smoothed using SPM99. From the subject responses, as recorded on the stimulus presentation $\mathrm{PC}$, two regressors for each run were constructed (see Materials and Methods and Fig. 4b).

Fig. 5 shows the SPM random-effects group analysis results at a significance level of 0.001 (uncorrected), overlaid to the SPM single subject brain. Two contrasts are shown in different colors: activation during task processing (blue-cyan) and pixel that are activated while answering (red-yellow). It can be seen that the SPM group analysis shows results similar to the selected single subject FCA results, i.e., the primary motor cortex is activated for answering only and not during task processing. Regions activated during answering included the primary somatomotoric area on the hemisphere contralateral to the button pressing, midline frontal areas, and bilateral insular regions. Additionally, activation in the ipsilateral central sulcus region was found. Activation during mental rotation was found bilaterally in the premotor and posterior parietal cortex.

\section{Discussion}

In this study we have used FCA as an exploratory tool to detect paradigm-related time courses in mental rotation fMRI data sets. In three subjects FCA has allowed the separation of distinct clusters corresponding either to visuospatial processing (i.e., mental rotation of the 3D cube stimuli) or to movement execution during answering (button pressing). Pixels of the first cluster are located in the parietal, occipital, and premotor cortex, as well as in anterior parts of the SMA. Pixels active during manual task response appeared in the primary somatomotor area (contralateral to the button-pressing hand) and more posterior parts of the SMA. It was, however, not possible to obtain similar cluster separations in all subjects. So the hypothesis that activity in M1 reflects motor activation necessary for task response rather than actual mental rotation was formed and tested using the parametric approach of SPM, where-in contrast to FCA-extensive a priori information is required. The statistical maps of the random effects group analysis confirmed that parietal and premotor areas are the main cortical regions used for mental rotation.

SPM analysis also confirms the hypothesis raised by FCA that M1 is not involved in spatial transformations of cube stimuli. Moreover, the fact that SMA is activated during task responding while more rostral mesial areas are recruited during task processing strengthens Wexler's thesis that mental rotation recruits motor planning and anticipation, but not the cortical and subcortical mechanisms responsible for movement execution (Wexler et al., 1998). It has been speculated that M1 activity, during mental rotation of figures of hands, was caused by the subjects visualizing themselves manipulating their right hands (Parsons et al., 1995; Kosslyn et al., 1998). Due to the stimuli used in our study, we cannot rule out that M1 might be involved in spatial processing of hand-like drawings. In particular, Kosslyn et al. (1998) explicitly demonstrated that ShepardMetzler figures do not activate M1. The situation for alphanumeric stimuli might also be different, as several studies either did find M1 activation (Tagaris et al., 1998; Vingerhoets et al., 2001) or denied primary motor cortex activity (Alivisatos and Petrides, 1997; Harris et al., 2000; Jordan et al., 2001). These results suggest that it may be the type of stimulus material used that determines whether M1 is used for task processing.

In conclusion, our study confirms that parietal and premotor areas are the main brain regions responsible for spatial transformations. Using Fuzzy Cluster Analysis we obtained an indicator that the primary motor cortex is not involved in task processing of the 3D cube comparison stimuli used in this study. Moreover, we have demonstrated that signal enhancement in M1 is a consequence of responding via button pressing rather than visuospatial processing. These results have been statistically verified with a random-effects group analysis over all 14 subjects. 


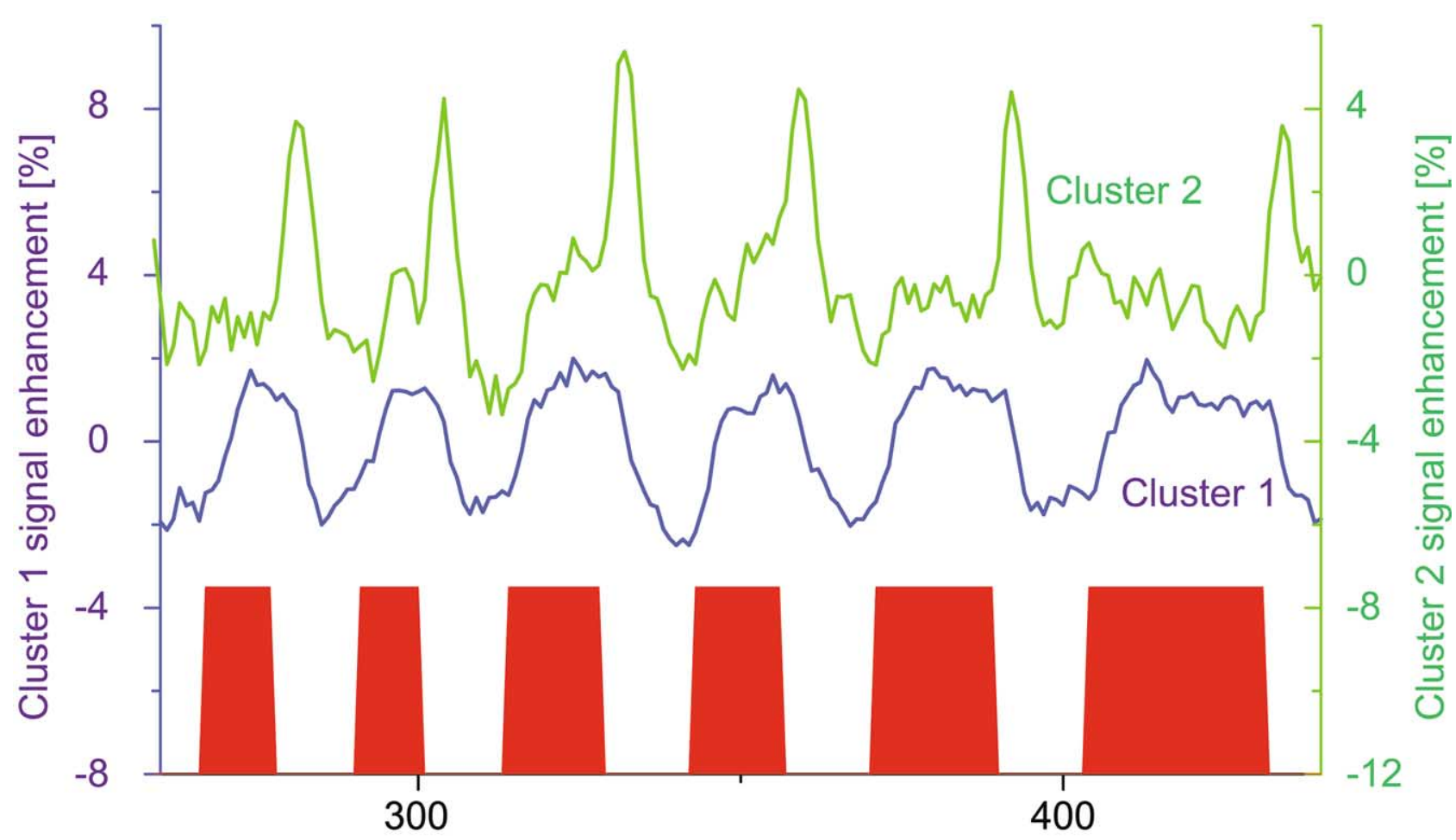

(a) image number

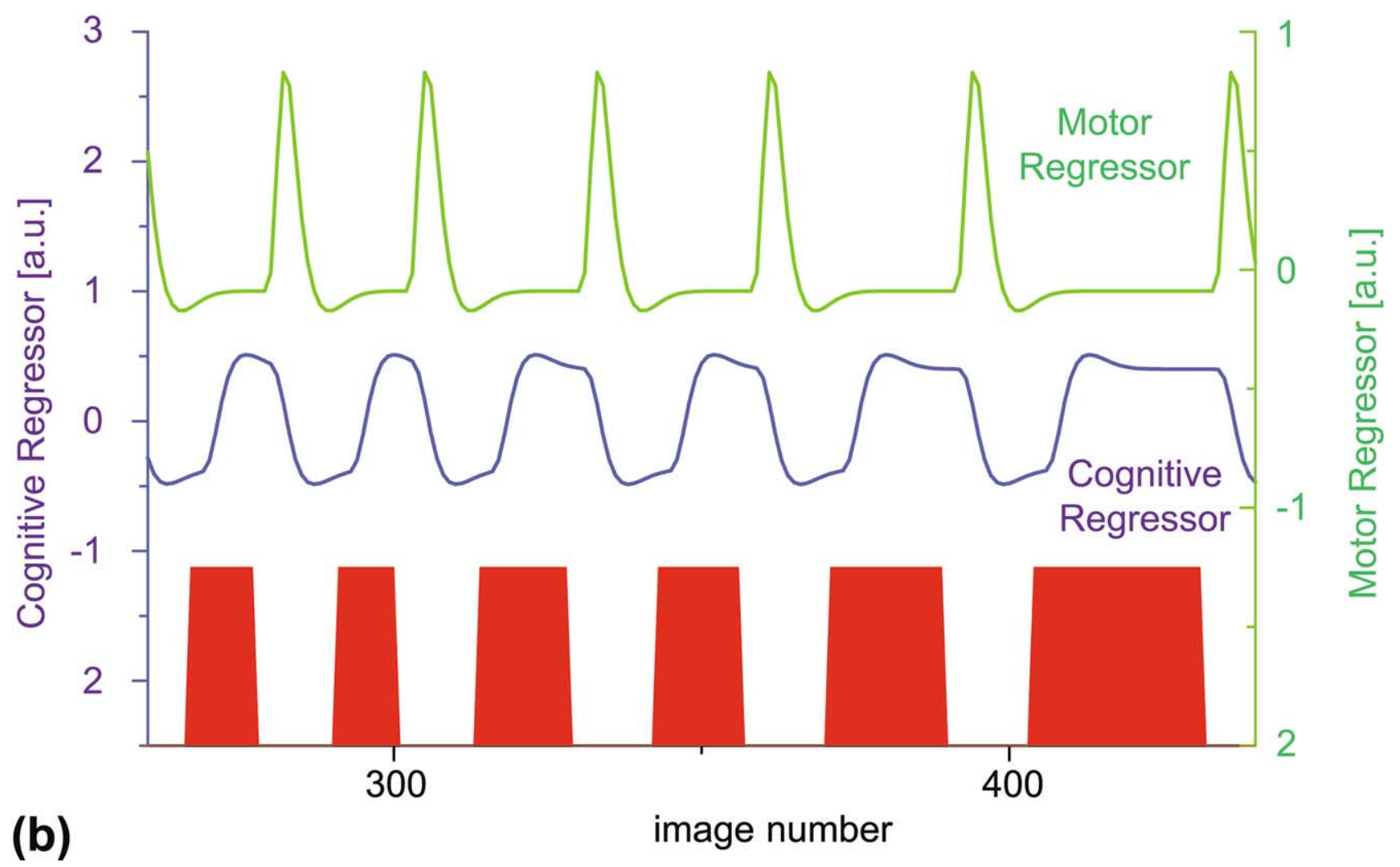

Fig. 4. (a) Enlarged part of the time courses given in Fig. 3. Again, cluster 2 shows signal enhancement at the end of the stimulus presentation period only. (b) Subject- and task-specific regressors used in the SPM analysis for the section shown in (a) and created according to the stimulus presentation protocol (see Materials and Methods). 


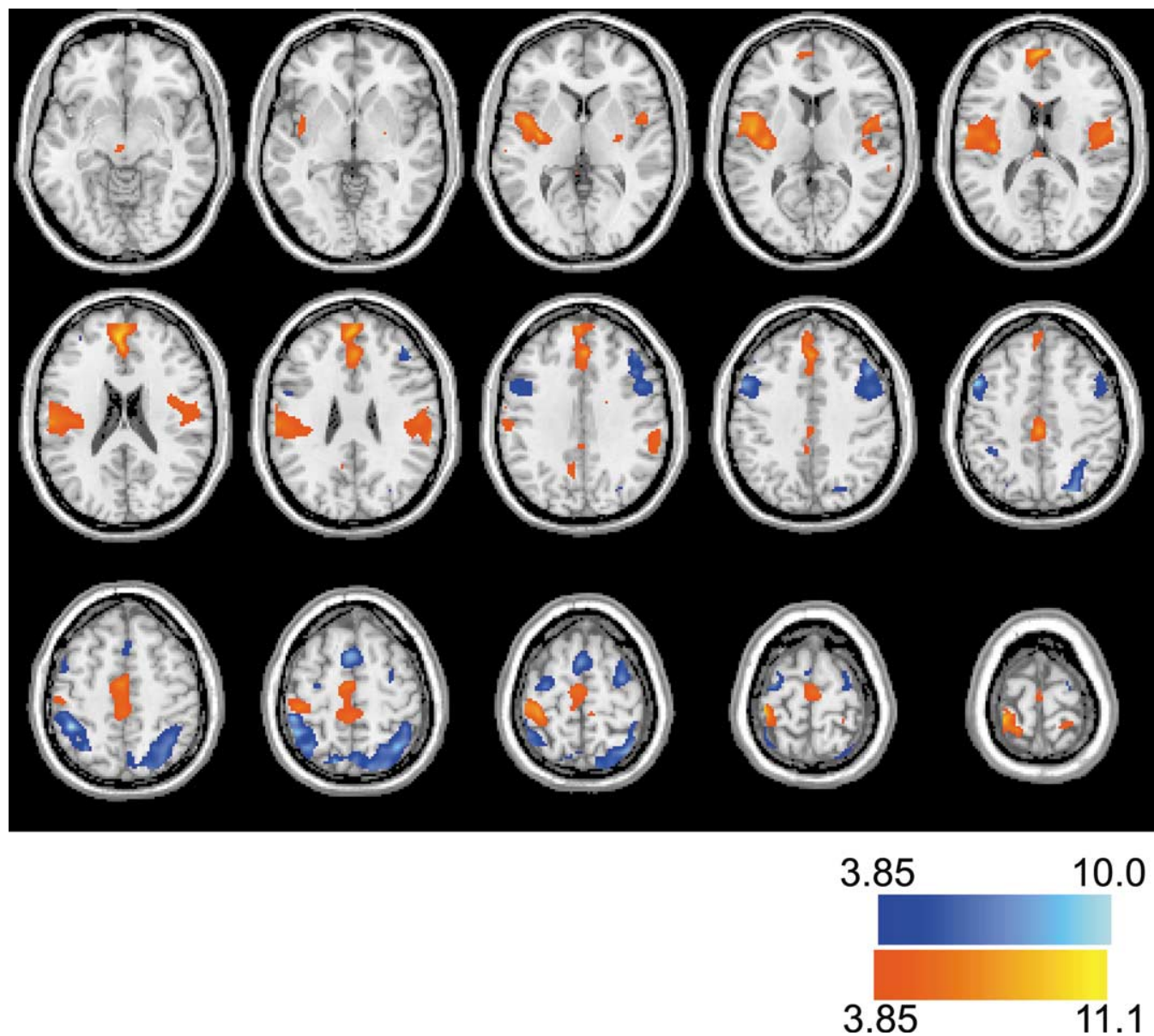

Fig. 5. Random-effects SPM group analysis results. Regions activated during stimulus presentation are colored blue to cyan; response activation is shown in red to yellow. Note the full separation of cognitive and response activation in the left frontoparietal region as well as the partitioning of activation in the frontomesial areas.

It remains unclear whether M1 might be active during mental rotation of stimuli different from the cubes used in this experiment.

This study was able to show the potential benefits of exploratory data analysis techniques such as FCA in examining functional networks in the brain by creating more specific prior knowledge for use in parametric analyses. Finally, in concordance with Lange et al. (1999) it seems that the combination of several analysis techniques may be advantageous to detect the full information available from fMRI experiments.

\section{Acknowledgments}

This work has been supported by the Jubiläumsfonds of the Austrian National Bank (7174, 8440). We gratefully acknowledge stimulating discussions with M. Jarmasz and R. Somorjai (Winnipeg, Canada). C.W. appreciates generous support by C. Suditu (Vienna, Austria).

\section{References}

Alivisatos, B., Petrides, M., 1997. Functional activation of the human brain during mental rotation. Neuropsychologia 35, 111.

Annett, M., 1985. Left, Right, Hand, and Brain. The Right Shift Theory, Erlbaum, London.

Backfrieder, W., Baumgartner, R., Samal, M., Moser, E., Bergmann, H., 1996. Quantification of intensity variations in functional MR images using rotated principal components. Phys. Med. Biol. 41, 1425.

Baumgartner, R., Windischberger, C., Moser, E., 1998. Quantification in functional magnetic resonance imaging: fuzzy clustering vs. correlation analysis. Magn. Reson. Imaging 16, 115.

Calhoun, V.D., Adali, T., Pearlson, G.D., Pekar, J.J., 2001. Spatial and temporal independent component analysis of functional MRI data containing a pair of task-related waveforms. Hum. Brain Mapp. 13, 43.

Carpenter, P.A., Just, M.A., Keller, T.A., Eddy, W., Thulborn, K., 1999. Graded functional activation in the visuospatial system with the amount of task demand. J. Cogn. Neurosci. 11, 9.

Cohen, M.S., Kosslyn, S.M., Breiter, H.C., DiGirolamo, G.J., Thompson, W.L., Anderson, A.K., Brookheimer, S.Y., Rosen, B.R., Belliveau, J.W., 1996. Changes in cortical activity during mental rotation. A mapping study using functional MRI. Brain 119, 89. 
Filzmoser, P., Baumgartner, R., Moser, E., 1999. A hierarchical clustering method for analyzing functional MR images. Magn. Reson. Imaging $17,817$.

Friston, K., Holmes, A., Worsley, K.J., Poline, J., Frith, C.D., Frackowiak, R.S., 1995. Statistical parametric maps in functional imaging: a general linear approach. Hum. Brain. Mapp. 2, 189.

Gittler, G., 1990. Dreidimensionaler Würfeltest (3DW). Beltz, Weinheim.

Goutte, C., Toft, P., Rostrup, E., Nielsen, F., Hansen, L.K., 1999. On clustering fMRI time series. NeuroImage 9, 298.

Harris, I.M., Egan, G.F., Sonkkila, C., Tochon-Danguy, H.J., Paxinos, G., Watson, J.D., 2000. Selective right parietal lobe activation during mental rotation: a parametric PET study. Brain 123, 65.

Jordan, K., Heinze, H.J., Lutz, K., Kanowski, M., Jancke, L., 2001. Cortical activations during the mental rotation of different visual objects. NeuroImage 13, 143.

Kosslyn, S.M., 1994. Image and Brain. MIT Press, Cambridge.

Kosslyn, S.M., DiGirolamo, G.J., Thompson, W.L., Alpert, N.M., 1998. Mental rotation of objects versus hands: neural mechanisms revealed by positron emission tomography. Psychophysiology 35, 151.

Lamm, C., Windischberger, C., Leodolter, U., Moser, E., Bauer, H., 2001. Evidence for premotor cortex activity during dynamic visuospatial imagery from single-trial functional magnetic resonance imaging and event-related slow cortical potentials. NeuroImage 14, 268.

Lange, N., Strother, S.C., Anderson, J.R., Nielsen, F.A., Holmes, A.P., Kolenda, T., Savoy, R., Hansen, L.K., 1999. Plurality and resemblance in fMRI data analysis. NeuroImage 10, 282.

McKeown, M.J., Jung, T.P., Makeig, S., Brown, G., Kindermann, S.S., Lee, T.W., Sejnowski, T.J., 1998. Analysis of fMRI data by blind separation into independent spatial components. Hum. Brain Mapp. 6, 160.

Moser, E., Diemling, M., Baumgartner, R., 1997. Fuzzy clustering of gradient-echo functional MRI in the human visual cortex. Part II: Quantification. J. Magn. Reson. Imaging 7, 1102.

Moser, E., Baumgartner, R., Barth, M., Windischberger, C., 1999. Explorative signal processing in functional MR Imaging. Int. J. Imag. Syst. Tech. 10, 166.

Parsons, L.M., Fox, P.T., Downs, J.H., Glass, T., Hirsch, T.B., Martin, C.C., Jerabek, P.A., Lancaster, J.L., 1995. Use of implicit motor imagery for visual shape discrimination as revealed by PET. Nature 375, 54.

Prinz, W., 1997. Perception and action planning. Eur. J. Cog. Psych. 9, 129.

Rasch, G., 1980. Probabilistic Models for Some Intelligence and Attainment Tests. Univ. of Chicago Press, Chicago.
Richter, W., Andersen, P.M., Georgopoulos, A.P., Kim, S.G., 1997. Sequential activity in human motor areas during a delayed cued finger movement task studied by time-resolved fMRI. NeuroReport 8, 1257.

Richter, W., Somorjai, R., Summers, R., Jarmasz, M., Menon, R.S., Gati, J.S., Georgopoulos, A.P., Tegeler, C., Ugurbil, K., Kim, S.G., 2000. Motor area activity during mental rotation studied by time-resolved single-trial fMRI. J. Cogn. Neurosci. 12, 310.

Scarth, G., McIntyre, M., Wowk, B., Somorjai, R., 1995. Detection of novelty in functional images using fuzzy clustering. Proc. SMR Nice 1, 238.

Shepard, R.N., Metzler, J., 1971. Mental rotation of three-dimensional objects. Science 171, 701.

Sychra, J.J., Bandettini, P.A., Bhattacharya, N., Lin, Q., 1994. Synthetic images by subspace transforms. I. Principal components images and related filters. Med. Phys. 21, 193.

Tagaris, G.A., Kim, S.-G., Strupp, J.P., Andersen, P., Ugurbil, K., Georgopoulos, A.P., 1997. Mental rotation by functional magnetic resonance imaging at high field (4 Tesla): performance and cortical activation. J. Cogn. Neurosci. 9, 419.

Tagaris, G.A., Richter, W., Kim, S.-G., Pellizzer, G., Andersen, P., Ugurbil, K., Georgopoulos, A.P., 1998. Functional magnetic resonance imaging of mental rotation and memory scanning: a multidimensional scaling analysis of brain activation patterns. Brain Res. Brain Res. Rev. $26,106$.

Vingerhoets, G., Santens, P., Van Laere, K., Lahorte, P., Dierckx, R.A., De Reuck, J., 2001. Regional brain activity during different paradigms of mental rotation in healthy volunteers: a positron emission tomography study. NeuroImage 13, 381.

Vingerhoets, G., de Lange, F.P., Vandemaele, P., Deblaere, K., Achten, E., 2002. Motor imagery in mental rotation: an fMRI study. NeuroImage $17,1623$.

Weimer, W.B., 1997. A conceptual framework for cognitive psychology: motor theories of the mind, in: Shaw, R., Bransford, J.D. (Eds.), Perceiving, Acting and Knowing, Erlbaum, Hillsdale, NJ.

Wexler, M., Kosslyn, S.M., Berthoz, A., 1998. Motor processes in mental rotation. Cognition 68, 77.

Windischberger, C., Moser, E., 2000. Spatial resolution in echo planar imaging: shifting the acquisition window in k-space. Magn. Reson. Imaging 18, 825 .

Woods, R.P., et al., 1998. Automated image registration: II. Intersubject validation of linear and nonlinear models. J. Comput. Assist. Tomogr. 22,153

Yoshino, A., Inoue, M., Suzuki, A., 2000. A topographic electrophysiologic study of mental rotation. Brain Res. Cogn. Brain Res. 9, 121. 